\title{
Rainbow medicine - supporting the needs of lesbian, gay, bisexual and trans patients
}

\author{
Author: Justin Varney ${ }^{\mathrm{A}}$
}

Over the last 50 years there have been huge societal shifts regarding sexuality, gender identity and sexual orientation. Homosexuality is no longer criminalised or pathologised and there are legal protections for trans individuals as well as for lesbian, gay and bisexual people. Against such societal progress some may ask 'why is it important for healthcare professionals to consider a patient's sexual orientation or gender identity at all'?

Although there has been legal progress, there were over 5,500 hate crimes reported to the police in $2014-15^{1}$ with sexual orientation attributed as the motivating factor, and a further 600 linked to being transgender. It is important to remember that although the first race equality legislation was passed in 1965 there were still over 42,000 race-related hate crimes recorded in 2014-15.

There is a significant and growing body of evidence ${ }^{2}$ that lesbian, gay, bisexual and trans (LGBT) people experience health inequalities across a range of conditions and diseases. Some of this is a reflection of the disproportionate distribution of infectious diseases such as HIV and hepatitis, some reflects increased health risk behaviours such as smoking, alcohol and drug misuse, and some is a result of worse mental health correlated with experiences of discrimination and marginalisation from family, friends and wider society. For gay and bisexual men there is growing recognition ${ }^{3}$ that these three domains of inequality may overlap and interact in an individual's life, although it is probably also true for lesbian and bisexual women and trans individuals.

The evidence base in the UK comes primarily from population level surveys commissioned by Stonewall and local authorities because sexual orientation has only been included in surveys by the Office of National Statistics since 2011. There is ongoing work to improve data collection on sexual orientation, particularly in NHS data, and a developing narrative about how to collect gender identity data at a population level.

However, even taking into account the variable sources, combined with the international research evidence base, the inequalities are stark:

$>$ Lesbian and gay youth are more likely to smoke and exhibit risky drinking behaviour than their heterosexual counterparts, and bisexual youth are more likely to smoke. ${ }^{4}$

Author: ${ }^{A}$ national lead for adult health and wellbeing, Public Health England, London, UK
> One in 20 gay and bisexual men in the UK are living with HIV; this rises to 1 in 11 in London. ${ }^{5}$

$>$ In a large US population survey, $46 \%$ of bisexual-identified female respondents reported experiencing rape (at some point in their life), compared with $17 \%$ of heterosexual women and $13 \%$ of lesbian women. ${ }^{6}$

$>$ Over half of lesbian, gay, bisexual, trans and questioning (LGBTQ) respondents (52\%) in the Youth Chances ${ }^{7}$ survey reported self-harming, either now or in the past. This compares to $35 \%$ of heterosexual non-trans young people in our sample and to a rate of $12 \%$ for this age-group that selfreported in a household survey by the NHS in 2007.

$>88 \%$ of trans people reported that they have experienced a mental health issue, over $37 \%$ have experienced physical threats or intimidation for being trans and 19\% have been hit or beaten for being trans. ${ }^{8}$

$>$ Older LGBT people are two and a half times as likely to live alone, twice as likely to be single and four and a half times as likely to have no children to call on in times of need as their heterosexual counterparts. ${ }^{9}$

The evidence ${ }^{10}$ also shows that for LGBT people who come from another minority identity, such as those living with a disability or from an ethnic minority group, the inequalities are even larger, probably because of the compound impacts of discrimination and marginalisation.

Sadly, health and social care services may be contributing to this discrimination and marginalisation. A 2015 YouGov survey ${ }^{11}$ of over 3,000 health and social care staff, commissioned by the charity Stonewall, found that just under a quarter of patient-facing staff had heard colleagues make negative comments about lesbian, gay or bisexual people while at work in the last 5 years; $20 \%$ had heard similar comments about trans people. Furthermore, $26 \%$ of lesbian, gay and bisexual staff reported having personally experienced bullying or poor treatment from colleagues because of their sexual orientation. This was against a context in which $72 \%$ had not received any training on the health needs of LGBT people or how to support them better in health and social care settings.

Training and knowledge are at the core of providing holistic patient care. Understanding the concepts of sexual orientation and gender identity are as fundamental as cultural competency for supporting ethnic minority patients. There is a lot of free training available, including free e-learning on sexual orientation on the Royal College of General Practitioners' platform commissioned by Public Health England and training 
commissioned by Gender Identity Research and Education Society (GIRES) on gender identity and gender reassignment pathways on the same platform. There is also face-to-face support available through the Pride In Practice programme delivered by the LGBT Foundation in Manchester, as well as factsheets and resources produced by the Department of Health; so the resources are there if we choose as clinicians to use them.

Making your practice more inclusive can be about small changes that can have a big impact; these include visible statements of diversity and equality inclusion. LGBT relevant material in the waiting room can provide non-verbal signs that your service is inclusive to patients and reassure all patients that you and your staff are willing and able to respond to diverse patient groups. Using inclusive language to ask about an individual's 'partner' rather than assuming an opposite gender 'husband' or 'wife' will help open opportunities for patients to disclose their sexual orientation and indicate that you as a practitioner are not assuming that everyone is heterosexual. Small changes in language and imagery can have a big impact on patients' perceptions of inclusion.

The research shows that LGBT people still feel that the NHS is failing to provide a safe and supportive space for them to receive care and that can lead to late presentation, treatment compliance issues and a lack of holistic and supportive care, which ultimately drives up costs and leads to worse clinical outcomes. We all have a duty as clinicians to educate ourselves to provide the best care we can for our patients, and this includes taking into account their sexual orientation and gender identity, not ignoring or dismissing it as irrelevant. Inclusive medicine is fundamentally good medicine and part of good medical practice.

\section{Conflicts of interests}

The author declares no conflicts of interests.

\section{References}

1 Corcoran H, Lader D, Smith K. Hate Crime, England and Wales, 2014/15. London: Home Office, 2015

2 Williams H, Varney J, Taylor J et al. The lesbian, gay, bisexual and trans public health outcomes framework companion document. London: Public Health England, 2013.

3 Public Health England. Promoting the health and wellbeing of gay, bisexual and other men who have sex with men: summary document. London: PHE, 2014.

4 Hagger-Johnson G, Taibjee R, Semlyen J et al. Sexual orientation identity in relation to smoking history and alcohol use at age 18/19: cross-sectional associations from the Longitudinal Study of Young People in England (LSYPE). BMJ Open 2013;3:e002810.

5 Skingsley A, Yin Z, Kirwan P et al. HIV in the UK - situation report 2015: incidence, prevalence and prevention. London: Public Health England, 2015.

6 Walters ML, Chen J, Breiding MJ. The national intimate partner and sexual violence survey: 2010 findings on victimization by sexual orientation. Atlanta: National Center for Injury Prevention and Control, Centers for Disease Control and Prevention, 2013.

7 METRO Youth Chances. Youth Chances summary of first findings: the experiences of LGBTQ young people in England. London: METRO, 2014

8 McNeil J, Bailey L, Ellis S, Morton J, Regan M. Trans mental health study 2012. Edinburgh: The Scottish Transgender Alliance, 2012.

9 Knocker S. The whole of me... Meeting the needs of older lesbians, gay men and bisexuals living in care homes and extra care housing. London: Age Concern, 2006.

10 Varney J. Minorities within minorities - the evidence base relating to minority groups within the LGB\&T community. Manchester: LGBT foundation, 2013.

11 Somerville C. Unhealthy attitudes: the treatment of LGBT people within health and social care services. London: Stonewall, 2015.

Address for correspondence: Dr J Varney, Public Health England, Skipton House, 80 London Road, London SE1 6LH, UK. Email: justin.varney@phe.gov.uk

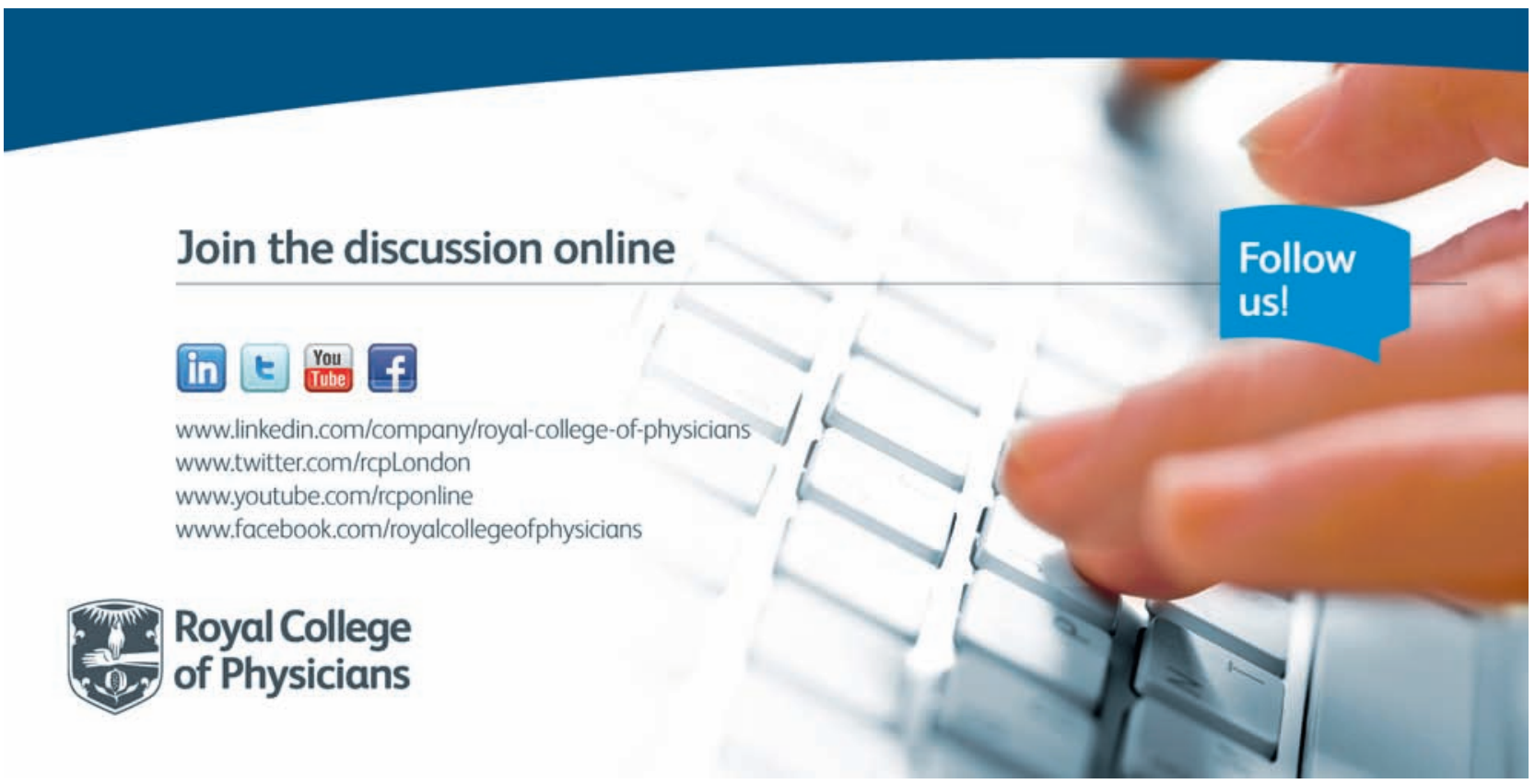

